\title{
Variations
}

Variations

Revue internationale de théorie critique

$22 \mid 2019$

Gorz, l'intempestif

\section{Changer la ville}

André Gorz et les communs urbains

\section{Francesco Brancaccio}

\section{(2) OpenEdition}

Journals

Édition électronique

URL : http://journals.openedition.org/variations/1170

DOI : $10.4000 /$ variations. 1170

ISSN : 1968-3960

Éditeur

Les amis de Variations

Référence électronique

Francesco Brancaccio, «Changer la ville», Variations [En ligne], 22 | 2019, mis en ligne le 04 mars

2019, consulté le 19 avril 2019. URL : http://journals.openedition.org/variations/1170 ; DOI : 10.4000/ variations. 1170

Ce document a été généré automatiquement le 19 avril 2019.

Les ami•e•s de Variations 


\title{
Changer la ville
}

\author{
André Gorz et les communs urbains
}

\section{Francesco Brancaccio}

1 Notre contribution ${ }^{1}$ repose sur la thèse suivante : si la sortie du capitalisme est plus que jamais actuelle, comme André Gorz l'affirme sans cesse dans ses derniers ouvrages, la réalisation de ce défi s'accompagne d'un changement radical concernant la façon dont l'espace urbain est pensé théoriquement, organisé matériellement et vécu collectivement. Autrement dit: la constitution «d'une autre économie, d'autres rapports sociaux, d'autres modes de vie et d'autres modes et moyens de production » (Gorz, 2008 : 36) va de pair avec l'affirmation d'une nouvelle politique de la ville (Gorz, 1997).

2 Notre propos est donc de suivre une voie encore peu explorée dans les études consacrées à l'œuvre de Gorz: sa contribution, directe ou indirecte, à une théorie critique de l'urbain. Des nombreuses études ${ }^{2}$ ont montré que l'espace, et en particulier l'espace urbain, aussi bien que les modalités de sa production et de son appropriation, constituent des enjeux stratégiques du point de vue de l'accumulation capitaliste mais aussi de celui des luttes qui s'y opposent.

3 Certes Gorz n'a jamais consacré un ouvrage spécifique à la question de l'urbain. De la lecture de ses travaux, il est toutefois possible de dégager de multiples suggestions pour enrichir la réflexion sur ce sujet. Dans ce but, il nous faudra mobiliser et relier plusieurs axes de recherche développés au cours des dernières décennies, sur les questions de l'économie de la connaissance, de l'écologie et de la nouvelle géographie urbaine. Pour ce faire nous rappellerons dans un première temps les traits saillants des nouvelles luttes urbaines en articulant cette thématique à deux axes de l'analyse de Gorz. Dans cette démarche, les premiers axes que nous évoquerons sont ceux avec lesquels Gorz a dialogué explicitement dans ses derniers écrits : l'écosophie de Félix Guattari et les thèses du capitalisme cognitif. Puis, nous ferons dialoguer l'œuvre de Gorz avec Henri Lefebvre ainsi que avec le marxisme géographique d'un auteur comme David Harvey. Enfin, nous tenterons de voir dans quelle mesure Gorz peut contribuer à la réflexion autour des communs urbains. 


\section{Gorz face aux luttes urbaines : la réappropriation de la ville à l'époque du néolibéralisme}

Il faut noter que les mouvements qui ont fait de la ville un enjeu central du rapport entre savoir et pouvoir constituent un facteur d'intérêt constant pour Gorz. Il suffit de songer, et non seulement en termes anecdotiques, à la sympathie politique qu'il exprimait au début des années 70 pour l'organisation italienne Lotta Continua, laquelle se ramifiait dans les quartiers populaires des principales villes italiennes, et dont l'un des slogans principa ux fut « Prendiamoci la città! » (« Prenons la ville !», Gianinazzi, 2016 : 144-145).

5 Pour commencer, essayons de faire nôtre le regard particulier que Gorz porte sur la réalité sociale. Dans l'un de ses livres le plus importants, L'Immatériel, tout en examinant l'organisation coopérative du travail et la force créative des communautés du logiciel libre - ce qu'il appelle les «dissidents du numérique» - Gorz déclare: "l'anarchocommunisme réellement existant du libre est une pratique, non un programme. $L a$ pratique est le programme. Le but n'est pas transcendant à l'action » (Gorz, 2003 : 113).

Ce même constat pourrait s'appliquer aux pratiques de commoning qui ont marqué et renouvelé la forme de certains mouvements sociaux à l'échelle mondiale. Au cours des dernières décennies, et avec une intensité croissante après le mouvement Occupy aux Etats Unis (2011) et celui des Indignados espagnols (2012), les luttes contre le néolibéralisme ont pris une dimension urbaine qui va bien au-delà de la simple occupation d'une place publique : ils ont expérimenté plus généralement des nouvelles formes d'appropriation et de socialisation de l'espace urbain que l'ont peut saisir à travers la notion de communs urbains. De nombreuses expériences confirment cette hypothèse : à la suite de ces mouvements on a vu apparaître des comités de quartier, des espaces de mutualisme, des usines régénérés, des tiers lieux de la production, des centres sociaux, des plateformes numériques alternatives, des expériences de syndicalisme social et autonome, etc.

7 Le " programme » d'une transformation globale de la réalité urbaine se situe d'emblée à l'intérieur et non à l'extérieur de ces expériences ; c'est-à-dire qu'il s'établit à partir de leur singularité et en fonction de la possibilité de les reproduire dans l'espace et dans le temps. L'urbain n'est pas tout simplement le «site» ou le «lieu» dans lequel ces pratiques se déploient. C'est le véritable enjeu stratégique de leur action. On pourrait considérer, en paraphrasant Gorz, que dans ces pratiques l'espace n'est pas transcendant à l'action, faisant lui-même l'objet d'une pratique collective. En effet, ces pratiques considèrent l'espace, et la manière dont celui-ci s'organise, comme une variable déterminante pour l'apparition de nouvelles façons de produire et d'organiser autrement le travail, mais aussi pour le surgissement d'usages alternatifs de la technique ou de nouveaux régimes juridiques qui remettent en question la logique propriétaire. Celles-ci se développent « à l'intérieur et contre » l'organisation socio-spatiale de l'urbain telle que le néolibéralisme l'a impulsée : l'urbanisation se present comme le vecteur central d'une nouvelle configuration globale des rapports de production au centre de la quelle se trouvent la rente et la finance. En outre, ils amènent sur le devant de la scène la nouvelle composition du travail vivant - ce que l'on peut appeler, à la suite de Negri et de Gorz, une « intelligence collective»-, caractérisée par la mobilisation des facultés cognitives, linguistiques, affectives et relationnelles des individus en tant que « forces productives immédiates ». Il convient d'ajouter qu'il s'agit de luttes exprimant un pouvoir constituant 
qui se déploie dans la création d'institutions autonomes et décentralisées, celles-ci cherchant à redéfinir les modes de production et de consommation ainsi que les mentalités et les styles de vie - comme c'est le cas pour les communs urbains.

\section{L'écologie politique et le travail de «production de soi »}

8 Les mouvements se déroulant dans le contexte urbain nous montrent le caractère ambivalent de celui-ci : en effet, il est à la fois l'espace où se déploient les nouvelles formes de domination et le champ privilégie pour l'essor de nouveaux territoires existentiels. Cette expression de Félix Guattari, avec lequel Gorz avait dialogué dans ses écrits sur l'écologie politique, exprime la nécessité de trouver une nouvelle approche pour aborder la question de la vie dans l'espace urbain. Dans un texte dédié aux "Pratiques écosophiques et à la restauration de la Cité subjective », et que Gorz cite dans Misères du Présent, Richesse du Possible, Guattari nous montre que l'écosophie porte aussi sur une écologie urbaine. A la différence des approches écologiques qui donnent à la nature le statut de sphère autonome à préserver de l'action extérieure de l'homme, la perspective écosophique de Guattari veut montrer que notre survie sur la Terre se verra menacée tant par la dégradation environnementale que par la désintégration du socius, du tissu de solidarité, et des modes de vies psychiques. Comme il l'affirme dans le texte déjà cité, «[..] on ne peut espérer recomposer une terre humainement sans la réinvention des finalités économiques et productives, des agencements urbains, des pratiques sociales, culturelles, artistiques et mentales " (Guattari, 2013a : 33). À cet égard, ce que Guattari définit comme "Cité subjective » est la figure matérielle et la détermination spatiale de son programme philosophique consistant en l'articulation de trois écologies : sociale, mentale et environnementale (Guattari, 1989). La réinvention de la ville, de ses formes, de ses flux, des territoires existentiels qui la composent, comme de ses « lignes de fuite ", équivaut à la recomposition d'un ensemble hétérogène de pratiques individuelles et sociales de "construction de soi-même " (Guattari, 2013b : 280) - c'est-à-dire, des pratiques visant à reconfigurer la globalité des relations humaines.

Pour Gorz comme pour Guattari, l'approche de l'écologie ne peut se réduire à la « défense de la nature ». Dans l'analyse que le premier fait des formes de dépossession et de privatisation, l'un des biens communs par excellence qui risque d'être détruit est le «monde vécu». La critique que Gorz adresse à ce qu'il définit comme l'écologie systémique est le corollaire de cette analyse : en réalité, une telle écologie est fondée sur la primauté incontestée de la science et, de la sorte, elle partage les mêmes postulats épistémiques qui fondent la rationalité capitaliste (Gollain, 2001 : 546). En effet, dans la critique que les deux auteurs font de cette rationalité, ce qui est en jeu dans l'écologie politique de Gorz comme dans la perspective écosophique de Guattari, c'est tout d'abord un déplacement de la valeur. Tandis que dans le capitalisme la valeur est enfermée à l'intérieur de la logique marchande et de l'équivalent général, dans les textes de Gorz et de Guattari sur l'écologie les figures de la valeur se démultiplient : " valeur de solidarité ", " valeur sociale ", " valeur intrinsèque ", " valeur esthétique ", " valeur écologique ». Les deux auteurs sont à la recherche de nouvelles modalités de valorisation dans le but de sortir de la logique marchande et de « la machine infernale d'une croissance économique aveuglement quantitative "(Guattari, 2013a: 33). Il s'agit de remettre en cause la séparation entre l'éthique et le politique pour comprendre les modifications de la 
production mais aussi et surtout la " production de soi " (Gorz) et les "modes de production de la subjectivité » (Guattari) deviennent un champ de bataille.

Or, pour Gorz, la réflexion philosophique sur la «production de soi » est décisive aussi bien pour préciser sa perspective écologique que la quête d'une nouvelle subjectivation politique du travail. C'est par là que sa réflexion rejoint la critique du capitalisme cognitif : si travailler c'est se produire (Gorz, 2003: 15), la production de soi doit être envisagée du point de vue d'une nouvelle configuration historique du conflit capital travail.

On pourrait évoquer trois raisons différentes pour lesquelles le concept de "production de soi » joue un rôle central dans la critique gorzienne du capitalisme cognitif.

Tout d'abord, cette expression synthétise les traits caractéristiques du travail vivant contemporain : coopération, action performative, facultés relationnelles et communicatives, capacité à répondre aux imprévus (Gorz, 2003 : 20). On peut préciser davantage cette idée en affirmant avec Paolo Virno que dans le capitalisme cognitif la production a intégré la plupart des prérogatives de l'action politique, en se présentant comme activité-sans-œuvre : "imprévisibilité, disposition à commencer quelque chose de nouveau, performances linguistique, habilité dans le choix entre des possibilité alternatives » (Virno, $2016: 222)$.

13 En second lieu, la production de soi désigne la base à partir de laquelle se redéfinissent la forme de l'entreprise comme ses stratégies d'exploitation. La réflexion de Gorz porte sur deux notions qui jouent un rôle central dans l'ordre du discours néolibéral et qui servent à identifier travail et entreprise : l'entrepreneur de soi-même et le capital humain (Gorz, 2001). En effet, l'entreprise déclenche une «mobilisation totale» afin de valoriser non seulement les résultats de la production de soi, mais la production de soi en tant que telle. C'est pourquoi l'exploitation prend l'apparence de l'auto-exploitation (Gorz, $2004: 206$ ). ${ }^{3}$

Il existe un troisième niveau auquel renvoie l'analyse de Gorz. Bien que le capitalisme opère une mobilisation totale des capacités corporelles et cognitives de l'individu, tout en le poussant à s'identifier à la forme de l'entreprise, la production de soi demeure toujours un terrain d'antagonisme :

«Cette coïncidence entre le travailleur et l'entreprise peut être vue sous deux faces. On peut y voir le signe que les capacités constitutives de la force de travail ne sont plus subsumables par le capital en tant qu'entité distincte, et que la nature de la production requiert maintenant un mode de coopération sociale auto-organisée, susceptible de déboucher tôt ou tard sur l'émancipation individuelle et collective des travailleurs » (Gorz, $2004: 207)$.

Si la productivité des entreprises dépend de plus en plus du " capital humain fixe », c'està-dire, des capacités de coopération, de communication et d'auto-organisation des individus, il faut se demander aussi à qui appartient ce capital humain et où a lieu son " accumulation primitive». Selon Gorz, il appartient à un fond culturel commun qui précède la valorisation de l'entreprise et qui n'est jamais totalement capturé par elle. C'est du côté de la résistance à la mobilisation totale mise en place par la figure néolibérale de l'entreprise que nous retrouvons le thème de l'appropriation de l'espace urbain.

«Un front de résistance totale à ce pouvoir s'ouvre. Il déborde nécessairement du terrain de la production de connaissances vers des nouvelles pratiques de vie, de consommation, d'appropriation collective des espaces communs et de la culture du quotidien. "Reclaim the Streets" en est une des expressions le plus réussies » (Gorz, $2004: 209)$. 


\section{Les nouvelles enclosures de l'urbain}

Poursuivant dans cette voie d'analyse, ce qui est en jeu dans le " passage d'échelle » opéré par l'urbanisation au niveau mondial au cours des dernières décennies c'est, tout d'abord, un bouleversement de ce que Gorz appelle la « culture du quotidien » et le « monde vécu ». C'est l'ensemble " des savoirs intuitifs, des savoir-faire vernaculaires, [...] des habitudes, des normes et des conduites allant de soi » (Gorz, $2008: 49)$ qui est investi par ces changements.

Or, la perte du monde vécu en tant que privatisation de savoirs correspond à la contradiction majeure que Gorz entrevoit dans la configuration actuelle des rapports sociaux de production ${ }^{4}$. En effet, le capitalisme cognitif, afin de soumettre la connaissance au circuit de la valorisation économique, fait recours à l'établissement de barrières artificielles à l'accès et de nouvelles enclosures de « biens » potentiellement cumulables à l'infini comme les savoirs, les connaissances et les informations. C'est ce que Gorz appelle création de positions de monopole par le biais du droit de propriété intellectuelle, du brand (le capital fictif et symbolique) et de nouveaux dispositifs techniques. Le dispositif propriétaire - se présentant comme un dominium directum sur la « chose » immatérielle joue ainsi un rôle décisif dans la redéfinition des rapports d'exploitation «lorsque l'accroissement de la rente devient le but déterminant de la politique des firmes » (Gorz, 2008: 33). Cette «logique rentière» du capitalisme cognitif (Vercellone, 2016), qui consiste à se placer à l'extérieur de l'organisation du processus productif, devient l'instrument privilégié pour contrôler et limiter le potentiel d'autonomie atteinte par la force travail cognitive dans l'organisation de la coopération sociale.

Du point de vue de la logique juridique, nous nous trouvons dans une situation opposée à celle décrite par la conception de la propriété de Locke: ce n'est pas la rareté d'une ressource - dans l'exemple de Locke, la terre - qui légitime la propriété (et en conséquence, la division sociale du travail), mais c'est l'imposition en amont d'une divisio primaeva (division originelle), au moyen du brevet, du copyright, de la marque, qui engendre une situation de pénurie artificielle d'une ressource abondante telle que la connaissance. Nous notons alors que la perspective gorzienne a entrevu avec une certaine anticipation ce qui aujourd'hui, dans le débat sur le commun et les biens communs, a été défini comme le «deuxième mouvement d'enclosures » (Boyle, 2008) ayant pour objet tant le savoir que le vivant et les institutions du welfare.

Ces trois éléments ont pour les travailleurs cognitifs une importance comparable, dans leur résistance actuelle au processus de privatisation, à celle eu jadis par les commons land à l'âge des premières enclosures. Il faut bien préciser que ce mouvement doit être entendu ici dans un double sens : le premier renvoie à la précarisation de la force de travail à travers la dépossession des moyens de production et de subsistance, et correspond à ce que Marx appelait la soumission formelle du travail au capital ; le second renvoie précisément à la dépossession des savoirs, ce qui correspond sur bien des aspects à ce que Marx appelait la logique de la soumission réelle du travail au capital. Ces deux dimension sont toujours étroitement combinées et adoptent, dans le capitalisme contemporain, une dimension spatiale qui trouve d'emblée dans la métropole - la nouvelle usine du post-fordisme (Negri, 2018) - le lieu central de la création de la valeur et de la mise en place de dispositifs de contrôle de la population. 
20 En effet, dans ce contexte de " mondialisation de l'urbainisation ", la ville, même si nous continuons à l'appeler ainsi, n'est plus la ville d'antan : elle est l'espace-temps de nouveaux mécanismes d'extraction de la valeur socialement produite, ainsi que de nouvelles formes d'expropriation des moyens de production et des savoirs. Un dynamique qui prend aujourd'hui la forme de la smart city reposant sur une gestion intégrée des services numériques et des big data gouvernée par le capitalisme des plateformes (Brancaccio et Vercellone, 2018, p. 51-70).

\section{Gorz et le droit à la ville : un dialogue avec Henri Lefebvre et David Harvey}

21 Nous tenterons maintenant d'entrelacer la perspective gorzienne avec le «droit à la ville », expression introduite par Henri Lefebvre en 1968 et qui est devenue le nomen communis d'un ensemble de pratiques et de mouvements d'appropriation de l'urbain.

Il convient de noter préalablement que dans ces mouvements, la référence au droit à la ville prend un double sens. D'une part, elle se présente sous la forme d'une dénonciation de quelque chose qui a été perdue, qui nous a été soustraite : le «droit » à une forme de vie collective - la vie urbaine - perturbée par le développement effréné de l'urbanisation et, plus récemment, par l'essor du capitalisme de plateforme et de l'ubérisation de la ville. ${ }^{5}$ En ce sens, le «droit à la ville » désigne, en négatif, la dégradation des conditions générales de vie dans les villes et les métropoles, la crise et la privatisation de l'espace public, la valorisation financière des espaces urbains par la rente immobilière, la mise en place de grands événements, l'expulsion des couches populaires des centres historiques transformés en quartiers vitrine ou en quartiers-Airbnb.

D'autre part, le « droit à la ville » est un claim ou, selon David Harvey, un « signifiant vide » évoqué par des mouvements urbains très différents les uns des autres (Harvey, 2011). Pour ne citer que quelques exemples: les mouvements Occupy de 2011 et Indignados de 2011 qui se sont caractérisés par l'appropriation d'un espace public par excellence comme l'est une place ; le mouvement pour la défense de Gezi Park à Istanbul ; le mouvement de 2013 au Brésil né pour revendiquer la gratuité des transports publics et pour contester le coût des structures dédiées à l'accueil des Championnats du monde de 2014. Cette séquence comprend également le mouvement "néomunicipaliste " né dans la ville de Naples, où une série d'occupations de bâtiments publics abandonnés dans le centre historique de la ville ont produit la naissance d'un nouveau «droit d'usage civique urbain " (dans le droit français, on dira un communaux urbain). Et encore on pourrait évoquer les comités de quartier qui luttent contre la spéculation urbaine d'Airbnb à Barcelone ainsi que dans de nombreuses autres villes européennes.

Effectivement l'un des enjeux de ces luttes est la recherche de modes alternatifs de socialisation de l'espace.

Si l'espace, comme l'affirme Lefebvre dans son ouvrage de 1974, La production de l'espace, est à la fois un "moyen de production » et " un produit ", sa socialisation devient un enjeu stratégique pour favoriser l'essor d'un autre mode de production. Ce que Lefebvre définit, dans le langage de son temps, comme "socialiste "- c'est-à-dire, fondé sur la participation effective et sur l'autogestion généralisée des lieux de production dans la ville. 
26 À cet égard, l'un des aspects le plus actuels dans l'analyse de Lefebvre est la distinction qu'il introduit, en mobilisant une lecture originale de Marx, entre la propriété e l'appropriation dans le contexte de la ville. Comme Lefebvre l'expose dans Du Rural à l'Urbain, en se référant à l'appropriation :

«il ne s'agit pas du tout de la propriété; il s'agit même de quelque chose de tout à fait différent; il s'agit du processus par lequel un individu ou groupe s'approprie, transforme en son bien quelque chose d'extérieur, de telle sorte que l'on peut parler d'un temps ou d'un espace urbain approprié au groupe qui a façonné la ville» (Lefebvre, $2001: 198$ ).

Si la propriété, la division juridique et spatiale du "mien" et du "tien", trouve son issue dans une domination destructrice de la nature, l'appropriation, en tant qu'acte collectif, est la capacité de transformer la réalité et de rétablir la primauté du droit à la vie urbaine.

Dans cette théorie renouvelée de l'aliénation dans l'espace urbain les pensées de Lefebvre et de Gorz se rapprochent et font résonner les mots du Marx des Manuscrits. L'abolition positive de la propriété privée, c'est-à-dire « l'appropriation sensible pour les hommes et par les hommes de la vie et de l'être humain» (Marx, 1972: 91) conduit à une modification des facultés sensibles de l'homme - facultés qui ne sont jamais des facultés individuelles mais communes - et de l'ensemble de ses relations avec le monde et la nature. Pour Lefebvre comme pour Gorz, le problème de l'aliénation ne renvoie pas, dans une perspective anthropologique, à la recherche d'une prétendue essence humaine perdue qui devrait être récupérée ou rétablie - tel est le cas des approches marxistes que Lefebvre définit comme "scolastiques » et "philosophantes». Pour Lefebvre l'aliénation est toujours pensée en fonction d'une possibilité inhérente à la praxis humaine de se désaliéner (Lefebvre, 1975 : 139).

Contre l'aliénation urbaine, Lefebvre propose le «droit à la ville». En tant qu'appel et exigence, il s'agit d'un projet politique à proprement parler : le droit à la ville est à la fois collectif et individuel. Il s'articule comme un "droit d'usage » et un "droit à l'œuvre ", c'est-à-dire comme possibilité d'utiliser et de s'approprier un bien ou un espace indépendamment de sa propriété. Son corollaire necessaire est la participation effective à la construction de l'espace urbain.

30 Le concept de droit à la ville a été également repris récemment par le géographe marxiste David Harvey. Il le fait dans le cadre d'une analyse basée sur le rôle crucial de l'urbanisation dans la reproduction des rapports de production capitalistes et sur les communs urbains comme forme par excellence de résistance à ce processus. Harvey a mis en évidence le double visage de l'accumulation du capital aujourd'hui : la plus classique «accumulation par exploitation" (accumulation by exploitation) se combine étroitement avec «l'accumulation par dépossession » (accumulation by dispossession), cette dernière reposant sur une logique essentiellement «prédatrice » et «extractive » des communs.

31 Comme il le souligne dans Villes Rebelles (Harvey, 2015), l'intérêt actuel pour les communs urbains correspond aux effets déstructurants de la vague de privatisations, à l'intensification des contrôles spatiaux et policiers ainsi que à la surveillance de la qualité de vie urbaine en général. En ce sens « le droit à la ville » se configure comme « le pouvoir de façonnement fondamental et radical sur les processus d'urbanisation, c'est-à-dire sur les manières dont nos villes sont sans cesse transformées » (Harvey, 2010 : 161).

32 Les villes sont caractérisées par des luttes continues pour l'appropriation collective de l'espace et contre son expropriation capitaliste, et c'est seulement au cours de ces luttes 
que la distinction entre ce qui est public et ce qui est commun peut émerger. Les espaces et les biens publics dans les villes ont toujours été le domaine du pouvoir d'État et de l'administration publique. Dans l'histoire de l'urbanisation, la réalisation de biens publics, tels que les égouts, la santé, l'éducation, les routes, ont été réalisés avec l'apport essentiel des capitaux publics. Dans les villes, théâtre de vigoureux conflits et de luttes de classes, les administrations urbaines ont été forcées de fournir des biens publics à la classe ouvrière urbanisée.

Or, l'impulsion qui fait que ces espaces, biens ou services publics deviennent communs, est une initiative politique " par le bas ", une initiative visant à se les approprier, c'est-àdire, visant à modifier collectivement leurs modes d'accès, d'usage et de gouvernance. Ainsi, une place ou une rue deviennent un bien commun parce que dans une lutte particulière, les modes de socialisation de l'espace sont changées. Harvey rapporte toute une séquence de places qui ont pris le statut de communs urbains : Syntagma à Athènes, Tahir au Caire, place de Catalunya à Barcelone, Puerta del Sol à Madrid, etc. De même, une école publique devient "commune" lorsque des comités entre parents et enseignants sont mis en place ; ou un hôpital, lorsque s'organisent des comités entre médecins et patients.

En résumé, l'apport de Harvey n'est pas seulement d'avoir encouragé la recherche sur un nouveau type de communs - les communs urbains - mais aussi d'avoir écarté une vision naturaliste à la Ostrom (1990) : les biens communs, en fait, ne préexistent pas au processus qui les génère. C'est toujours la mise en commun (commoning) qui fait d'un bien o d'un service, public ou privé, un bien ou un service commun. Ce qui importe, ce ne sont pas les caractéristiques intrinsèques d'un bien, mais les relations sociales qui modifient son statut et la manière dont il est utilisé.

\section{Notes pour un programme de recherche sur les communs urbains}

Si ses analyses ont renouvelé la perspective du droit à la ville, il est toutefois opportun d'en souligner certaines limites.

Une première limite est de caractère temporel. Dans l'analyse de Harvey, le commun semble émerger essentiellement comme une réaction aux pratiques prédatrices du capitalisme. Néanmoins, comme nous l'avons montré dans un livre dédié au Commun comme mode de production (Vercellone et alii, 2017) la caractérisation de la dynamique des communs urbains dans le temps et dans l'espace, mise en place par les commoners (Sultan 2017), naît d'un double mouvement à la fois offensif et défensif : offensif, car ils traduisent la dynamique endogène d'une économie fondée sur la connaissance et la force d'invention d'un "savoir vivant" d'une intelligence collective qui a pris son essor depuis la crise du fordisme et des institutions de la société disciplinaire ; défensif, car les communs représentent une forme de résistance aux nouvelles enclosures du savoir, du vivant et des institutions du welfare, qui ont trouvé dans l'espace urbain leur lieu de prédilection. En effet, les processus néolibéraux de corporization, de propertization et de commodification se heurtent à des contre-tendances puissantes et celles-ci trouvent dans les communs l'incarnation d'une alternative au monopole du binôme public-privé qui a marqué de son sceau l'âge du capitalisme industriel. 

bornée par son caractère spatial. Notre hypothèse est qu'il est nécessaire d'étendre l'objet «communs urbains » des espaces publics aux lieux de la production. Il en résulte une définition extensive des communs urbains, qui englobe la multiplicité des expériences dans le domaine de l'émancipation du travail comme dans celui de la réappropriation des moyens de production, des savoirs et des produits. Les urban commons, à notre avis, constituent à la fois une déclinaison essentielle d'une nouvelle " économie du commun » et une catégorie juridique émergente (droit d'usage civique, nouveaux communaux, community land trust, etc.). Par définition, leur construction sociale est indissociable des espaces urbains dans lesquels ils s'installent et qu'ils modifient. Sur ce point, la contribution de Gorz est fondamentale, s'il est vrai, comme le souligne Carlo Vercellone (2018), que dans la troisième phase de sa vie, Gorz élabore un nouveau projet de société visant la réappropriation des moyens de production et l'émancipation dans le travail, un projet qui prend la forme des communs de l'écologie et de la connaissance.

Dans Misères du Présent, Richesse du Possible, Gorz consacre une partie à l'impératif de « changer la ville ». La ville qu'imagine Gorz est une ville polycentrique, décentralisée et intelligible, qui encourage en son sein les pratiques d'auto-production et d'autoapprentissage ainsi que le partage et l'échange de services et des savoirs. Au centre de cette ville se trouvent les " cercles de coopération ", ou comme il les désignera plus tard, dans Ecologica (Gorz, 2008: 41), " les ateliers communaux ». Ce sont des lieux d'autoorganisation pour les producteurs associés et ils sont basés essentiellement sur deux éléments : d'une part, l'établissement de circuits monétaires locaux alternatifs visant à affirmer, contre la valeur d'échange, la valeur sociale ; d'autre part, l'utilisation du potentiel offert par l'informatisation qui ne cessera pas d'accroître la capacité des réseaux de coopération - ce que Gorz nomme comme l'appropriation collective des nouvelles technologies (Gorz, $1997: 160$ ). Dans ces cercles et dans leur éventuelle mise en réseau s'amorce, selon Gorz, une « critique pratique du travail emploi ». Chaque cercle est un collectif dont les membres s'approprient le travail, sa répartition, sa division et la définition, l'acquisition, la diffusion des savoirs, compétences, techniques » (Gorz, 1997 : 163). Ce projet autogestionnaire est étroitement lié à la réflexion actuelle sur les biens communs urbains et à la possibilité que la logique du copyleft - à travers la diffusion du paradigme de l'Internet des objets et des makerspaces - puisse également affecter la production matérielle. De nombreuses expériences menées durant ces dernières années ont commencé à être expérimentées dans ce domaine associant les sphères de la production immatérielle et matérielle. À ce propos, Gorz évoque la manière dont les ateliers communaux interconnectés pourraient combiner deux dimensions de l'utopie des communs : le partage, sans entraves juridiques et spatiales, de tous les «inputs » immatériels (le savoir, les informations, le logiciel, les données) grâce à l'extension de la logique du copyleft ; la constitution au niveau local d'une économie de la contribution où la participation à la production et la distribution des produits serait régie selon les principes d'une démocratie radicale.

Ainsi, après avoir présenté la proposition d'un revenu social de base et l'augmentation du temps disponible " non comme des réducteurs mais comme des multiplicateurs d'activité » (Gorz, 1997 : 149), Gorz entame cette réflexion dans le but de penser l'exode de la société du travail et de la marchandise : « En changeant la ville, nous fournirons un levier au changement de société et au changement de la manière dont les personnes vivent leurs rapports et leur inhérence au monde » (Gorz, 1997 : 151). Or, il s'agit ici précisément 
d'une reconquête du temps et de l'espace vécu, et c'est en cela que Gorz rejoindre Lefebvre : «L'usage réapparaît en conflit aigu avec l'échange dans l'espace, car il implique "appropriation" et non "propriété". Or, l'appropriation elle-même implique du temps et des temps, un rythme ou des rythmes, des symboles et une pratique » (Lefebvre, 2000 : 411).

En effet, la politique de la ville proposée par Gorz se fonde, comme chez Lefebvre, sur la conviction selon laquelle il faut émanciper la ville de la valeur d'échange et au-delà la libérer de la logique marchande et de la "servitude volontaire ». Et tout cela n'est possible qu'en remettant au centre la question d'un droit d'usage tout en le renvoyant à sa vocation originaire, celle de favoriser la conjonction entre la poiesis (production) et la praxis (action politique). Aussi cette conception de l'usage au cœur du droit à la ville chez Lefebvre et de la politique de la ville chez Gorz, devient-il le levier conceptuel indispensable pour penser une sortie du capitalisme fondé sur le commun et un nouveau projet autogestionnaire.

\section{BIBLIOGRAPHIE}

Boyle J., 2008, The Public Domain. Enclosing the Commons of the Mind, New Haven \& London : Yale University Press.

Brancaccio F., Vercellone C., 2018, in Vercellone C. (dir.), Brancaccio F., Giuliani A., Puletti F., Rocchi G., Vattimo P., 2018, Data-driven disruptive commons-based models, DECODE project, European Union's Horizon 2020, grant agreement no. 732546 : https://decodeproject.eu/ publications/data-driven-disruptive-commons-based-models , p. 51-70.

Brossat I., 2018, Airbnb ou la ville uberisée, Paris : La ville brûle Ed.

Gianinazzi W., 2016, André Gorz, une vie, Paris : La Découverte.

Gollain F., 2011, «L'apport d'André Gorz au débat sur le capitalisme cognitif », Revue du MAUSS, $1, \mathrm{n}^{\circ} 35$, p. 541-558.

Gorz A., 2008, Ecologica, Paris : Galilée.

Gorz A., 2004, «Économie de la connaissance, exploitation des savoirs. Entretien avec Carlo Vercellone et Yann Moulier Boutang », Multitudes, 1, n 15, p. 205-216. DOI 10.3917/ mult.015.0205.

Gorz A., 2003, L'Immatériel. Connaissance, valeur et capital, Paris : Galilée.

Gorz A., 2001, « La personne devient une entreprise ». Note sur le travail de production de soi, Revue du MAUSS, 2, n 18, p. 61-68.

Gorz A., 1997, Misères du présent, richesse du possible, Paris : Galilée.

Guattari F., 2013a, « Pratiques subjectives et restauration de la cité subjective », in Guattari F., Qu'est-ce que l'écosophie?, textes présentés par Stépane Nadaud, Paris : Lignes, 2013, p. 31-58.

Guattari F., 2013b, « Les modes de production de subjectivité », in Guattari F., Qu'est-ce que l'écosophie?, textes présentés par Stépane Nadaud, Paris : Lignes, 2013, p. 277-284. 
Guattari, F., 1989, Les Trois Écologies, Paris : Galilée.

Harvey D., 2015, Villes Rebelles. Du droit à la ville à la révolution urbaine, Paris : Buchet-Chastel.

Harvey D., 2011, Le capitalisme contre le droit à la ville. Néoliberalisme, urbanisation, résistances, Paris : Editions Amstérdam.

Harvey D., 2010, Géographie et capital. Vers un matérialisme historico-géographique, Paris : Editions Syllepse.

Lefebvre H., 2009, Le droit à la ville, 3ème édition, Paris : Anthropos.

Lefebvre H., 2000, La production de l'espace, 4ème édition, Paris : Anthropos.

Lefebvre H., 1975, L’idéologie structuraliste, Paris : Seuil.

Marx K., 2018, Manuscrits de 1857 - 1858 dits “Grundrisse”, Réimpression photonumérique de l'édition Jean-Pierre Lefebvre, Paris : Editions Sociales.

Marx K., 1972, Manuscrits de 1844, Paris : Editions Sociales.

Mezzadra S., Neilson B., 2013, Border as Method, or, the Multiplication of Labor, Durham North Carolina : Duke University Press

Morozov E., Bria F., 2018, Rethinking The Smart City. Democratizing Urban Technology, New York : Rosa Luxemburg Stiftung, New York Office.

Negri A., 2018, From The Factory To The Metropolis, Translated by Ed Emery, Cambridge, New York : Polity Press.

Ostrom E.,1990, Governing the Commons: The Evolution of Institutions for Collective Action, Cambridge : Cambridge University Press.

Sassen S., 2018, Cities in a World Economy, Fourth Edition, Thousand Oaks (Californie) et Londres : Pine Forge Press.

Soja E.W., 1989, Postmodern Geographies, Londres-New York : Verso.

Sultan F., 2017, Commoner, in Cornu M., Orsi F., Rochfeld J. (dir.), 2017, Dictionnaire des biens communs, Paris : PUF.

Vercellone C. 2018, Le travail entre aliénation et émancipation. Une introduction au marxisme hétérodoxe d'André Gorz, in Granjon F., (dir.) Matérialismes, culture et communication, Tome II, Paris : Presses des Mines, à paraître.

Vercellone C., Brancaccio F., Giuliani A., Vattimo P., 2017, Il Comune come modo di produzione. Per una critica dell'economia politica dei beni comuni, Verone : Ombre Corte.

Vercellone C., 2016, «Les institutions du welfare comme enjeu de la crise. Vers un welfare du commun », Contretemps : Revue de critique communiste, Textuel, <halshs-01335203>.

Vercellone C., 2012, L'analyse "gorzienne" de l'évolution du capitalisme, in Fourel C. (dir.), 2012, André Gorz. Un penseur pour le XXI siècle, Paris : La Découverte, p. 79-100.

Virno P., 2016, «Virtuosité et Révolution. Théorie politique de l'exode », in Virno P., L'usage de la vie et autres sujets d'inquiétude, Paris: Editions de l'éclat, 2016, p. 219-274. 


\section{NOTES}

1. Cette contribution est la réélaboration de l'intervention que j'ai tenue à l'occasion de la journée d'étude Gorz l'intempestif qui a eu lieu le 4 mai 2018 à la Halle Saint-Pierre à Paris. La journée a été organisée par Alexander Neumann, Carlo Vercellone et moi-même.

2. Outre les travaux d'Henri Lefebvre et de David Harvey auxquels nous nous référerons dans cette contribution, voir aussi : Sassen, 2018; Mezzadra et Neilson, 2013; Soja, 1989.

3. Gorz nous donne une figure spatiale de cette tendance, aujourd'hui réalisée dans des lieux comme la Silicon Valley : les villes entreprise (company towns), créées de toutes pièces par les grandes firmes américaines, où le « lieu de travail y est aménagé de manière à être un lieu de vie ", où « toute la vie fait partie du travail, le travail est toute la vie », et où « la firme offre le bonheur dans la servitude volontaire » (Gorz, $2004: 208$ ).

4. Avec l'avènement du capitalisme cognitif, les savoirs se formalisent en connaissance et sont placés au cœur du processus de valorisation, en tant que «force productive immédiate ». Sur ce point, l'analyse de Gorz croise celle du Marx du Fragment sur les Machines des Grundrisse (2018), pour qui les savoirs ne sont pas seulement des savoirs morts - la science et la technologie incorporés dans des machines en tant que capital fixe, mais aussi et surtout des savoirs vivants incarnés dans une "intelligence collective » et qui débordent cette incorporation (Vercellone : 2012).

5. Sur l'ubérisation de la ville : Brossat, 2018. Pour une critique de la smart city : Morozov et Bria, 2018.

\section{INDEX}

Mots-clés : Politique de la ville, écologie politique, Felix Guattari, André Gorz, David Harvey, Henri Lefebvre

\section{AUTEUR}

\section{FRANCESCO BRANCACCIO}

Francesco Brancaccio est Attaché temporaire d'enseignement et de recherche à l'UFR Culture et Communication de l'Université Paris 8. Il est doctorant au Cemti-Critic. 